\title{
Dressing cosets revisited
}

\author{
R. Squellari ${ }^{a, b}$ \\ anstitut de mathématiques de Luminy, \\ 163, Avenue de Luminy, \\ 13288 Marseille, France \\ ${ }^{b}$ Laboratoire de Physique Théorique et des Hautes Energies \\ CNRS, Unité associée URA 280 \\ 2 Place Jussieu, F-75251 Paris Cedex 05, France
}

\begin{abstract}
We present an alternative algebraic derivation of the dual pair of nonlinear $\sigma$-models based on the 'dressing cosets' extension of the Poisson-Lie $T$-duality [2]. Then we generalize the result to dual pairs of Lagrangians not considered in [2]. Our generalization turns out to incorporate the dualisable models constructed by Sfetsos in 5 .
\end{abstract}




\section{Introduction}

Poisson-Lie $T$-duality [1] establishes a dynamical equivalence of certain nonlinear $\sigma$-models, the target manifolds of which are a Poisson-Lie group $G$ and its dual Poisson-Lie group $\tilde{G}$, respectively. Those models admit a generalization [2] for which the targets of the mutually dual $\sigma$-models are respectively the spaces of the dressing orbits $F \backslash G$ and $F \backslash \tilde{G}$ where $F$ is certain (isotropic) subgroup of the common Drinfeld double $D$ of $G$ and $\tilde{G}$. The most economic way to describe the Lagrangian dynamics of the models on $F \backslash G$ and $F \backslash \tilde{G}$ is in terms of $\sigma$-models living apparently on the targets $G$ and $\tilde{G}$ but admitting a local dressing gauge symmetry with the gauge group being the current group with values in $F[2$. We remark that this description is the dressing analogue of the usual description of the Lagrangian dynamics of the standard gauged $G / H$ WZW $\sigma$-models in terms of the $G$ target only (cf. Eq. (2.8) of [3]). In that case the gauge group $H$ acts on the apparent target $G$ in the adjoint way.

The brief and somewhat sketchy derivation of the dynamics of the dressing cosets presented in [2] was based on the methods of symplectic geometry namely on the symplectic reduction of certain coadjoint orbit of the centrally extended loop group of the Drinfeld double $D$. In this article, we furnish a more algebraic derivation of the second-order action of the dressing cosets found in [2]. Futhermore, we show how our new algebraic derivation leads to a generalization of the dressing cosets construction of Ref.[2]. We identify explicitly the actions of the generalized dressing cosets and confirm by direct calculation their gauge invariance. Finally we show that the models constructed by Sfetsos in [5] fit in our generalized construction.

In Section 2, we introduce our new algebraic calculus through the example of the standard Poisson-Lie T-duality and, in Section 3, we use it to derive the actions of dressing coset models of Ref.[2]. In Section 4, by still using the same algebraic method, we obtain new dual pairs of models which were not considered in 2] and we call them the generalized dressing cosets. In Section 5, we check explicitly the gauge symmetry of all dressing cosets models (standard or generalized), and in Section 6 we show that the $\sigma$-models found by Sfetsos [5] can be interpreted as the generalized dressing cosets. We end up with a short Section 7 containing the conclusions.

\section{Preliminaries}

Let us introduce few preliminary concepts which will be common for all particular cases studied in this article. Recall that a Drinfeld double is a 
$2 n$-dimensional Lie group $D$ such that its Lie algebra $\mathcal{D}$ contains two $n$ dimensional subalgebras $\mathcal{G}$ and $\hat{\mathcal{G}}$ which are complementary, i.e. $\mathcal{G} \cap \hat{\mathcal{G}}=\{0\}$, and which are isotropic with respect to a non-degenerate invariant bilinear form on $\mathcal{D}$ denoted $\langle. \mid$.$\rangle , i.e. \langle\mathcal{G} \mid \mathcal{G}\rangle=\langle\hat{\mathcal{G}} \mid \hat{\mathcal{G}}\rangle=0$. In this paper we shall consider only the so-called perfect Drinfeld doubles for which every element $l \in D$ can be written in a unique way as $l=g \hat{h}$ for some $g \in G$ and some $\hat{h} \in \hat{G}$ where $G$ and $\hat{G}$ are the subgroups of $D$ which integrate the Lie subalgebras $\mathcal{G}$ and $\hat{\mathcal{G}}$ of $\mathcal{D}$.

The crucial structural ingredient of all models presented in this paper will be certain linear operator $R: \hat{\mathcal{G}} \rightarrow \mathcal{G}$ and its adjoint $R^{*}: \hat{\mathcal{G}} \rightarrow \mathcal{G}$ with respect to the bilinear form $\langle. \mid$.$\rangle . In fact, we shall see that the properties of the kernels$ of $R$ and $R^{*}$ will classify the different classes of Poisson-Lie $\sigma$-models. Indeed, the case $\operatorname{Ker} R=\operatorname{Ker} R^{*}=\{0\}$ and $\operatorname{Ker}\left(R+R^{*}\right)=\{0\}$ underlies the standard Poisson-Lie T-duality considered in [1]; the case $\operatorname{Ker} R=\operatorname{Ker} R^{*}=\{0\}$ and $\operatorname{Ker}\left(R+R^{*}\right) \neq\{0\}$ will lead to the dressing cosets introduced in [2]; the general case is obtained when $\operatorname{Ker} R=\operatorname{Ker} R^{*} \neq\{0\}$ while $\operatorname{Ker}\left(R+R^{*}\right) \neq$ $\{0\}$. The subcase of general case for which $\operatorname{Ker} R=\operatorname{Ker} R^{*}=\operatorname{Ker}\left(R+R^{*}\right) \neq$ $\{0\}$ will give the models introduced by Sfetsos [5].

As a warm-up to help us to set up the language, we consider the simplest case of the standard Poisson-Lie T-duality: models with $\operatorname{Ker} R=\operatorname{Ker} R^{*}=$ $\operatorname{Ker}\left(R+R^{*}\right)=\{0\}$.

First order action: Parametrize a two-dimensional world-sheet by a spacelike coordinate $\sigma$ and a time-like coordinate $\tau$ and consider a $D$-valued field $l(\sigma, \tau)$. For the first order action governing the dynamics of these fields we take

$$
\mathcal{S}(l)=\int\left(\frac{1}{2}\left\langle\partial_{\tau} l l^{-1} \mid \partial_{\sigma} l l^{-1}\right\rangle+\frac{1}{12} d^{-1}\left\langle d l l^{-1} \wedge\left[d l l^{-1} \wedge d l l^{-1}\right]\right\rangle+\frac{1}{2} K\left(\partial_{\sigma} l l^{-1}\right)\right),
$$

where we omit the measure $d \tau d \sigma$ of integration for the rest of the article. In the action (11), $d l l^{-1}=\partial_{\tau} l l^{-1} d \tau+\partial_{\sigma} l l^{-1} d \sigma$ denotes the pull-back of the right-invariant Maurer-Cartan form from $D$ to the world-sheet, and $K$ is a certain quadratic form defined on $\mathcal{D}$. Before expliciting the expression of the quadratic form $K$, we need the following theorem:

Theorem 1. Let $R: \hat{\mathcal{G}} \rightarrow \mathcal{G}$ and $R^{*}: \hat{\mathcal{G}} \rightarrow \mathcal{G}$ such that $\operatorname{Ker} R=\operatorname{Ker} R^{*}=\{0\}$ and $\operatorname{Ker}\left(R+R^{*}\right)=\{0\}$, then there exists an unique pair of linear operators $\alpha_{ \pm}: \mathcal{D} \rightarrow \hat{\mathcal{G}}$ such that any $X \in \mathcal{D}$ can be decomposed as:

$$
X=\alpha_{+}(X)+\alpha_{-}(X)+R \alpha_{+}(X)-R^{*} \alpha_{-}(X) .
$$


Proof. Let us take $X \in \mathcal{D}$, then we know that there exists an unique decomposition $X=x+a$ where $x \in \mathcal{G}$ and $a \in \hat{\mathcal{G}}$. In our analyse we suppose the relation (2) verified, then by projecting on the algebras $\hat{\mathcal{G}}$ and $\mathcal{G}$ we obtain the system:

$$
\left\{\begin{array} { l } 
{ a = \alpha _ { + } ( X ) + \alpha _ { - } ( X ) } \\
{ x = R \alpha _ { + } ( X ) - R ^ { * } \alpha _ { - } ( X ) }
\end{array} \text { , or } \left\{\begin{array}{l}
\left(R+R^{*}\right) \alpha_{-}(X)=R a-x \\
\left(R+R^{*}\right) \alpha_{+}(X)=R^{*} a+x
\end{array} .\right.\right.
$$

Since in our case $\operatorname{Ker}\left(R+R^{*}\right)$ is trivial, we find explicitly the expression of the $\alpha_{ \pm}$operators:

$$
\left\{\begin{array}{l}
\alpha_{-}(X)=\left(R+R^{*}\right)^{-1}(R a-x) \\
\alpha_{+}(X)=\left(R+R^{*}\right)^{-1}\left(R^{*} a+x\right)
\end{array} .\right.
$$

Thus we have found the unique pair of operators $\alpha_{ \pm}$, which fit in the decomposition (2).

We define a quadratic form $K$ on $\mathcal{D}$ by:

$$
K(X)=2\left\langle\alpha_{+}(X) \mid R \alpha_{+}(X)\right\rangle+2\left\langle\alpha_{-}(X) \mid R \alpha_{-}(X)\right\rangle .
$$

This definition completes the description of the first order action (11). However, it is perhaps not evident at the first sight that the quadratic form $K$ coincides with the quadratic form appearing in Ref.[1, [6]. Let us see that this is indeed true. We observe that the subspaces $\mathcal{R}=\{\gamma+R \gamma, \gamma \in \hat{\mathcal{G}}\}$ and its orthogonal complement $\mathcal{R}^{\perp}=\left\{\gamma-R^{*} \gamma, \gamma \in \hat{\mathcal{G}}\right\}$ are $n$-dimensional. Moreover we see that $\operatorname{Ker}\left(R+R^{*}\right)=\{0\}$ implies $\mathcal{R} \cap \mathcal{R}^{\perp}=\{0\}$, hence $\mathcal{R} \oplus \mathcal{R}^{\perp}=\mathcal{D}$. Recall that the quadratic form $K^{\prime}$ appearing in Ref. [6] has two eigenvalues \pm 1 corresponding to the eigenspaces $\mathcal{R}$ and $\mathcal{R}^{\perp}$ respectively. The result is that we obtain the expression of $K^{\prime}(X)$ for all $X \in \mathcal{D}$ as:

$$
\begin{aligned}
K^{\prime}(X)= & \left\langle\alpha_{+}(X)+R \alpha_{+}(X) \mid \alpha_{+}(X)+R \alpha_{+}(X)\right\rangle \\
& -\left\langle\alpha_{-}(X)-R^{*} \alpha_{-}(X) \mid \alpha_{+}(X)-R^{*} \alpha_{-}(X)\right\rangle \\
= & 2\left\langle\alpha_{+}(X) \mid R \alpha_{+}(X)\right\rangle+2\left\langle\alpha_{-}(X) \mid R \alpha_{-}(X)\right\rangle=K(X) .
\end{aligned}
$$

We can easily determine the equation of motion verified by the $l(\sigma, \tau) \in D$ field, thus the extremalization of the action (11) gives:

$$
\partial_{\tau} l l^{-1}+\alpha_{+}\left(\partial_{\sigma} l l^{-1}\right)-\alpha_{-}\left(\partial_{\sigma} l l^{-1}\right)+R \alpha_{+}\left(\partial_{\sigma} l l^{-1}\right)+R^{*} \alpha_{-}\left(\partial_{\sigma} l l^{-1}\right)=0 .
$$


Poisson-Lie $\sigma$-models: Because the double $D$ is perfect, we can represent any field $l(\sigma, \tau) \in D$ as $l(\sigma, \tau)=g(\sigma, \tau) \hat{h}(\sigma, \tau)$ with $g(\sigma, \tau) \in G$ and $\hat{h}(\sigma, \tau) \in$ $\hat{G}$. Using this parametrization and the Polyakov-Wiegmann formula [7] the action (11) becomes:

$$
\mathcal{S}(g, \hat{h})=\int\left\langle g^{-1} \partial_{\tau} g \mid \partial_{\sigma} \hat{h} \hat{h}^{-1}\right\rangle+\frac{1}{2} K\left(\partial_{\sigma} g g^{-1}+\operatorname{Ad}_{g} \partial_{\sigma} \hat{h} \hat{h}^{-1}\right) .
$$

Applying the decomposition (21) on $X=\partial_{\sigma} l l^{-1}=\partial_{\sigma} g g^{-1}+\operatorname{Ad}_{g} \partial_{\sigma} \hat{h} \hat{h}^{-1}$, the action (8) becomes:

$$
\mathcal{S}(g, \hat{h})=\int\left\langle\partial_{\tau} g g^{-1} \mid \alpha_{+}+\alpha_{-}\right\rangle+\left\langle\alpha_{+} \mid R \alpha_{+}\right\rangle+\left\langle\alpha_{-} \mid R \alpha_{-}\right\rangle,
$$

where we denoted $\alpha_{ \pm}\left(\partial_{\sigma} l l^{-1}\right)=\alpha_{ \pm}$and used Eq (5). Now we define two operators $J(\hat{J})$ on $\mathcal{D}$, which project to $\mathcal{G}(\hat{\mathcal{G}})$ with the kernel $\hat{\mathcal{G}}(\mathcal{G})$ respectively. Let us project the decomposition:

$$
\partial_{\sigma} l l^{-1}=\partial_{\sigma} g g^{-1}+\operatorname{Ad}_{g} \partial_{\sigma} \hat{h} \hat{h}^{-1}=\alpha_{+}+\alpha_{-}+R \alpha_{+}-R^{*} \alpha_{-},
$$

on the subspaces $\hat{\mathcal{G}}$ and $\mathcal{G}$ :

$$
\begin{aligned}
\alpha_{+}+\alpha_{-} & =\hat{J} \operatorname{Ad}_{g} \hat{J} \partial_{\sigma} \hat{h} \hat{h}^{-1} \\
R \alpha_{+}-R^{*} \alpha_{-} & =\partial_{\sigma} g g^{-1}+J \operatorname{Ad}_{g} \hat{J} \partial_{\sigma} \hat{h} \hat{h}^{-1} .
\end{aligned}
$$

In Eq.(11), the operator $\hat{J} \operatorname{Ad}_{g} \hat{J}: \hat{\mathcal{G}} \rightarrow \hat{\mathcal{G}}$ is invertible, which gives:

$$
\partial_{\sigma} \hat{h} \hat{h}^{-1}=\hat{J} \operatorname{Ad}_{g^{-1}} \hat{J}\left(\alpha_{+}+\alpha_{-}\right) .
$$

Inserting relation (13) in Eq.(12), we obtain:

$$
\begin{aligned}
R \alpha_{+}-R^{*} \alpha_{-} & =\partial_{\sigma} g g^{-1}+\Pi^{g *}\left(\alpha_{+}+\alpha_{-}\right) \quad \text { or, equivalently: } \\
\partial_{\sigma} g g^{-1} & =\left(R+\Pi^{g}\right) \alpha_{+}-\left(R+\Pi^{g}\right)^{*} \alpha_{-} .
\end{aligned}
$$

Here

$$
\begin{aligned}
& \Pi^{g}=J_{\operatorname{Ad}_{g} J \operatorname{Ad}_{g^{-1}} \hat{J} \text { and }} \\
& \Pi^{g *}=J_{\operatorname{Ad}_{g} \hat{J} \operatorname{Ad}_{g^{-1}} \hat{J}}
\end{aligned}
$$

are two $g$-dependent operators from $\hat{\mathcal{G}}$ into $\mathcal{G}$. By the way, it turns out that $\Pi^{g}$ defines the so called Poisson-Lie structure 11 on $G$.

\footnotetext{
${ }^{1}$ More precisely, the Poisson bracket on the group $G$ can be expressed in term of the operator $\Pi^{g}$ as $\{f, g\}=\left\langle\nabla f \mid \Pi^{g} \nabla g\right\rangle$. Here $(f, g)$ are two arbitrary fonctions of $G$ and $\nabla \in \hat{\mathcal{G}}$ is defined by $\langle\nabla \mid x\rangle=\nabla^{x}$ where $\nabla^{x}$ is a differential operator generating the left action of $\mathcal{G}(x \in \mathcal{G})$ on $G$.
} 
Consider now any skew symetric $g$ dependent operator $M^{g}: \hat{\mathcal{G}} \rightarrow \mathcal{G}$, i.e. verifying $\left\langle M^{g} x \mid y\right\rangle=-\left\langle x \mid M^{g} y\right\rangle, \forall(x, y) \in \hat{\mathcal{G}}$. The action (9) can be rewritten in a more complicated but usefull way as:

$$
\mathcal{S}(g, \hat{h})=\int\left\langle\partial_{\tau} g g^{-1} \mid \alpha_{+}+\alpha_{-}\right\rangle+\left\langle\alpha_{+} \mid\left(R+M^{g}\right) \alpha_{+}\right\rangle+\left\langle\alpha_{-} \mid\left(R+M^{g}\right) \alpha_{-}\right\rangle .
$$

If moreover $R+M^{g}$ is invertible, then the action(16) can be written in even more complicated way as:

$$
S(g, \hat{h})=-\frac{1}{2} \int\left\langle Y \mid\left(R+M^{g}\right) Y\right\rangle+\left\langle Z-\partial_{\tau} g g^{-1}\right|\left(R+M^{g}\right)^{-1}\left(Z+\partial_{\tau} g g^{-1}\right\rangle,
$$

where

$$
\begin{aligned}
Y & =\left(R+M^{g}\right)^{-1}\left[\left(R+M^{g}\right) \alpha_{+}+\left(R+M^{g}\right)^{*} \alpha_{-}-\partial_{\sigma} g g^{-1}\right] \\
Y & =2 \alpha_{+}-\left(R+M^{g}\right)^{-1} \partial_{+} g g^{-1} \\
Z & =\left(R+M^{g}\right) \alpha_{+}-\left(R+M^{g}\right)^{*} \alpha_{-} .
\end{aligned}
$$

Here $\xi_{ \pm}=\tau \pm \sigma$ and $\partial_{ \pm}=\partial_{\tau} \pm \partial_{\sigma}$ are the light-cone coordinates.

Why this apparent complication? Because, we want to study the variation problem, in which $\hat{h}$ varies while $g$ remains fixed. Indeed, it turns out that there is a choice of the skew adjoint operator $M^{g}$ for which the $Z$ part of the action (17) depends only on $g$, and all dependence on $\hat{h}$ is hidden only in the quantity $Y$. This choice is simply $M^{g}=\Pi^{g}$, because of the relation (15), we see that $Z=\partial_{\sigma} g g^{-1}$ and is $\hat{h}$ independent. Futhermore, the following relation (Eq.(21) ) between $Y$ and $\partial_{\sigma} \hat{h} \hat{h}^{-1}$ becomes invertible (i.e. one can express $\partial_{\sigma} \hat{h} \hat{h}^{-1}$ in term of $Y$ ) since it only involves the invertible operators $\left(R+\Pi^{g}\right),\left(R+R^{*}\right)$ and $\hat{J} \operatorname{Ad}_{g} \hat{J}$. The relation relating the $Y$ variable to $\partial_{\sigma} \hat{h} \hat{h}^{-1}$, is given by:

$$
\begin{aligned}
Y= & 2\left(R+R^{*}\right)^{-1}\left(R+\Pi^{g}\right)^{*} \hat{J} \operatorname{Ad}_{g} \hat{J} \partial_{\sigma} \hat{h} \hat{h}^{-1} \\
& +2\left(R+R^{*}\right)^{-1} \partial_{\sigma} g g^{-1}-\left(R+\Pi^{g}\right)^{-1} \partial_{+} g g^{-1},
\end{aligned}
$$

Thus in the minimization of the action (17) with $M^{g}=\Pi^{g}$, the variation of the $n$ components of the variable $\partial_{\sigma} \hat{h} \hat{h}^{-1}$ is equivalent to the variation of the $n$ variables in $Y$.

Consequently, in the action (17) the quadratic term containing $Y$ simply disappears upon the variation since there is no linear $Y$ term. Upon replacing $Z$ by $\partial_{\sigma} g g^{-1}$ we conclude that the $\hat{h}$-variation leads to the standard PoissonLie second order action[1]:

$$
\mathcal{S}(g)=\frac{1}{2} \int d \xi_{+} d \xi_{-}\left\langle\partial_{-} g g^{-1} \mid\left(R+\Pi^{g}\right)^{-1} \partial_{+} g g^{-1}\right\rangle, \quad g \in G
$$


Duality: Where is the duality in all the formalism which we have just presented ? To see it, we define a linear operator $\hat{R}: \mathcal{G} \rightarrow \hat{\mathcal{G}}$ given by

$$
\hat{R}=R^{-1},
$$

and consider its adjoint $\hat{R}^{*}: \mathcal{G} \rightarrow \hat{\mathcal{G}}$. Obviously Eq.(23) implies that $\operatorname{Ker} \hat{R}=$ $\operatorname{Ker} \hat{R}^{*}=\{0\}$ and $\operatorname{Ker}\left(\hat{R}+\hat{R}^{*}\right)=\{0\}$. From the Theorem 1, we conclude that there exists an unique set of linear operators $\hat{\alpha}_{ \pm}: \mathcal{D} \rightarrow \mathcal{G}$ such that for any $X \in \mathcal{D}$ we can decompose it as:

$$
X=\hat{\alpha}_{+}(X)+\hat{\alpha}_{-}(X)+\hat{R} \hat{\alpha}_{+}(X)-\hat{R}^{*} \hat{\alpha}_{-}(X) .
$$

Now we can easily verify that the quadratic form $K$ on $\mathcal{D}$ defined by (15) can be rewritten as:

$$
K(X)=2\left\langle\hat{\alpha}_{+} \mid \hat{R} \hat{\alpha}_{+}\right\rangle+2\left\langle\hat{\alpha}_{-} \mid \hat{R} \hat{\alpha}_{-}\right\rangle .
$$

Indeed, Eq.(25) comes from the fact that the subspaces $\mathcal{R}$ and $\mathcal{R}^{\perp}$ can be expressed in term of the $\hat{R}$ operators:

$$
\mathcal{R}=\{\hat{\gamma}+\hat{R} \hat{\gamma}, \hat{\gamma} \in \mathcal{G}\} \text { and } \mathcal{R}^{\perp}=\left\{\hat{\gamma}-\hat{R}^{*} \hat{\gamma}, \hat{\gamma} \in \mathcal{G}\right\}
$$

Mimicking the same steps as previously, where we vary $h(\sigma, \tau)$ in the dual ansatz $l(\sigma, \tau)=\hat{g}(\sigma, \tau) h(\sigma, \tau) \in D$ with $\hat{g}(\sigma, \tau) \in \hat{G}$ and $h(\sigma, \tau) \in G$, we thus obtain the dual action on $\hat{G}$ :

$$
S(\hat{g})=\frac{1}{2} \int d \xi_{+} d \xi_{-}\left\langle\partial_{-} \hat{g} \hat{g}^{-1} \mid\left(R^{-1}+\hat{\Pi}^{\hat{g}}\right)^{-1} \partial_{+} \hat{g} \hat{g}^{-1}\right\rangle, \quad \hat{g} \in \hat{G},
$$

where $\hat{\Pi}^{g}: \mathcal{G} \rightarrow \hat{\mathcal{G}}$ the Poisson-Lie operator on $\hat{\mathcal{G}}$.

We point out already in this section, that more general models appear when the kernel of the operator $R$ or/and the kernel of the sum $R+R^{*}$ is/are not trivial. In the Section 4 and 5 , we will consider all possibilities just mentioned, and relate them to models already considered in Refs. [2], and [5]. We note that the case where both kernels are not trivial has not been considered before and will constitute the principal original result of this paper.

\section{Models with $\operatorname{Ker} R=\operatorname{Ker} R^{*}=\{0\}$ and $\operatorname{Ker}(R+$ $\left.R^{*}\right) \neq\{0\}$}

In this part we suppose that $\operatorname{Ker}\left(R+R^{*}\right) \neq\{0\}$ while still $\operatorname{Ker} R=\operatorname{Ker} R^{*}=$ $\{0\}$ and we show that this generalization leads again to a duality between 
two second order $\sigma$-models living on different target spaces. Moreover, under some further conditions on the operator $R$, the second order $\sigma$-models develop an intriguing gauge symmetry which is a novel aspect comparing to the case $\operatorname{Ker}\left(R+R^{*}\right)=\{0\}$.

As before the main structural ingredients of our story are the two operators $R: \hat{\mathcal{G}} \rightarrow \mathcal{G}, R^{*}: \hat{\mathcal{G}} \rightarrow \mathcal{G}$. Define $\hat{R}: \mathcal{G} \rightarrow \hat{\mathcal{G}}$ by the relation:

$$
\hat{R}=R^{-1} \text {. }
$$

It follows that the $\operatorname{kernel} \operatorname{Ker}\left(\hat{R}+\hat{R}^{*}\right)$ can be simply expressed as:

$$
\operatorname{Ker}\left(\hat{R}+\hat{R}^{*}\right)=R \operatorname{Ker}\left(R+R^{*}\right) .
$$

First order action: Let us introduce the following linear subspaces of $\mathcal{D}$ :

$$
\begin{aligned}
\mathcal{R} & =\{\gamma+R \gamma, \gamma \in \hat{\mathcal{G}}\} \\
\mathcal{R}^{\perp} & =\left\{\gamma-R^{*} \gamma, \gamma \in \hat{\mathcal{G}}\right\} \\
\mathcal{F} & =\left\{\gamma+R \gamma, \gamma \in \operatorname{Ker}\left(R+R^{*}\right)\right\}=\left\{\gamma-R^{*} \gamma, \gamma \in \operatorname{Ker}\left(R+R^{*}\right)\right\}
\end{aligned}
$$

Note that $\mathcal{R}^{\perp}$ is orthogonal to $\mathcal{R}$ as the notation suggests and $\mathcal{F}$ is necessarily isotropic $(\langle\mathcal{F} \mid \mathcal{F}\rangle=0)$ since $\mathcal{F}=\mathcal{R} \cap \mathcal{R}^{\perp}$. We shall soon show (cf. Theorem 2) that the orthogonal complement $\mathcal{F}^{\perp}$ of $\mathcal{F}$ in $\mathcal{D}$ can be written as $\mathcal{F}^{\perp}=$ $\mathcal{R}+\mathcal{R}^{\perp}$. We stress, however, that this is just the sum and not the direct sum of linear subspaces since $\mathcal{R} \cap \mathcal{R}^{\perp} \neq\{0\}$.

Consider a $D$-valued field $l(\sigma, \tau)$ and an $\mathcal{F}$-valued field $\Lambda(\sigma, \tau)$. Now write the first order action as:

$\mathcal{S}(l)=\frac{1}{2} \int\left\langle\partial_{\tau} l l^{-1} \mid \partial_{\sigma} l l^{-1}\right\rangle+\frac{1}{6} d^{-1}\left\langle d l l^{-1} \wedge\left[d l l^{-1} \wedge d l l^{-1}\right]\right\rangle+K\left(\partial_{\sigma} l l^{-1}\right)+\left\langle\Lambda \mid \partial_{\sigma} l l^{-1}\right\rangle$.

Note that the field $\Lambda$ is the Lagrange multiplier the variation of which imposes the constraint $\partial_{\sigma} l l^{-1} \in \mathcal{F}^{\perp}$.

Because of this new constraint, it is sufficient to define the quadratic form $K$ only on $\mathcal{F}^{\perp}$. For that we have to adapt the Theorem 1 to this new case.

Theorem 2. There exists a pair of linear operators $\alpha_{ \pm}: \mathcal{F}^{\perp} \rightarrow \hat{\mathcal{G}}$ such that $X \in \mathcal{F}^{\perp}$ can be decomposed as:

$$
X=\alpha_{+}(X)+\alpha_{-}(X)+R \alpha_{+}(X)-R^{*} \alpha_{-}(X) .
$$

Proof. Let us consider the subspace of $\mathcal{D}$ formed by the sum $\mathcal{R}+\mathcal{R}^{\perp}$, and let us take any element $X \in \mathcal{R}+\mathcal{R}^{\perp}$. Then, with the definitions (29) (30), there exists couples $(\alpha, \beta) \in \hat{\mathcal{G}}$ such that:

$$
X=\alpha+R \alpha+\beta-R^{*} \beta .
$$


Let us show that $\mathcal{R}+\mathcal{R}^{\perp}$ is orthogonal to $\mathcal{F}$, for that consider any element $\gamma \in \operatorname{Ker}\left(R+R^{*}\right)$ and calculate the following expression for $X \in \mathcal{R}+\mathcal{R}^{\perp}$ :

$$
\begin{aligned}
\langle X \mid \gamma+R \gamma\rangle & =\left\langle\alpha+R \alpha+\beta-R^{*} \beta, \gamma+R \gamma\right\rangle \\
& =\langle\alpha+\beta \mid R \gamma\rangle+\left\langle R \alpha-R^{*} \beta \mid \gamma\right\rangle \\
& =\langle\alpha+\beta \mid R \gamma\rangle+\left\langle\alpha \mid R^{*} \gamma\right\rangle-\langle\beta \mid R \gamma\rangle \\
& =\left\langle\alpha \mid\left(R+R^{*}\right) \gamma\right\rangle \\
& =0 .
\end{aligned}
$$

The result is that $\mathcal{R}+\mathcal{R}^{\perp} \subset \mathcal{F}^{\perp}$. Let us analyze the dimension of the $\mathcal{R}+\mathcal{R}^{\perp}$ space, we have the equality:

$$
\begin{aligned}
\operatorname{dim}\left(\mathcal{R}+\mathcal{R}^{\perp}\right) & =\operatorname{dim} \mathcal{R}+\operatorname{dim} \mathcal{R}^{\perp}-\operatorname{dim}\left(\mathcal{R} \cap \mathcal{R}^{\perp}\right) \\
& =2 n-\operatorname{dim} \mathcal{F} .
\end{aligned}
$$

Denoting $\operatorname{Ker}\left(R+R^{*}\right)^{0}=\left\{x \in \mathcal{G} / \forall \gamma \in \operatorname{Ker}\left(R+R^{*}\right),\langle x \mid \gamma\rangle=0\right\}$ the annihilator of $\operatorname{Ker}\left(R+R^{*}\right)$ in $\mathcal{G}$, we consider any complement subspace $W$ of $\operatorname{Ker}\left(R+R^{*}\right)^{0}$ in $\mathcal{G}$, which means that we can write $W \oplus \operatorname{Ker}\left(R+R^{*}\right)^{0}=\mathcal{G}$ and that $\operatorname{dim} W=\operatorname{dim} \mathcal{F}$. Let us introduce the following subspace $W+\mathcal{F}^{\perp} \subset \mathcal{D}$, and take any non null $v \in W$. Then since $\operatorname{Ker}\left(R+R^{*}\right)^{0} \cap W=\{0\}$ there exists a $\gamma \in \operatorname{Ker}\left(R+R^{*}\right)$ such that $\langle v \mid \gamma+R \gamma\rangle=\langle v \mid \gamma\rangle \neq 0$, therefore $W \cap \mathcal{F}^{\perp}=\{0\}$. Consequently, $W+\mathcal{F}^{\perp}=W \oplus \mathcal{F}^{\perp}$ and we obtain for the dimensions $\operatorname{dim} \mathcal{F}^{\perp} \leq 2 n-\operatorname{dim} \mathcal{F}$. Moreover, we have $\mathcal{R}+\mathcal{R}^{\perp} \subset \mathcal{F}^{\perp}$ which implies with (35) that $\operatorname{dim} \mathcal{F}^{\perp}=\operatorname{dim}\left(\mathcal{R}+\mathcal{R}^{\perp}\right)$ and the equality $\mathcal{F}^{\perp}=\mathcal{R}+\mathcal{R}^{\perp}$. Therefore, for all $X \in \mathcal{F}^{\perp}$ there exists a (non-unique) couple $(\alpha, \beta) \in \hat{\mathcal{G}}$ such that $X=\alpha+R \alpha+\beta-R^{*} \beta$, and by identifying $\alpha \equiv \alpha_{+}(X)$ and $\beta \equiv \alpha_{-}(X)$ we obtain the proof.

We can now define the quadratic form $K$ on $\mathcal{F}^{\perp}$ in terms of the operators $\alpha_{ \pm}$:

$$
K(X)=2\left\langle\alpha_{+}(X), R \alpha_{+}(X)\right\rangle+2\left\langle\alpha_{-}(X), R \alpha_{-}(X)\right\rangle, \forall X \in \mathcal{F}^{\perp} .
$$

Remark: We stress that the choice of $\alpha_{ \pm}$is not unique contrary to the case of the Section 2. Indeed, if we take any linear operator $\Phi: \mathcal{F}^{\perp} \rightarrow$ $\operatorname{Ker}\left(R+R^{*}\right)$, the decomposition (33) remains unchanged by the tranformation $\alpha_{ \pm} \rightarrow \alpha_{ \pm} \pm \Phi$. Inspite of this ambiguity, we can check that the quadratic form $K(36)$ is defined on $\mathcal{F}^{\perp}$ unambiguously, i.e. its value on $X \in \mathcal{F}^{\perp}$ does not depend on the choice of $\alpha_{ \pm}$.

We can also easily determine the equations of motion verified by the $l$ field. 
By taking into account the constraint $\partial_{\sigma} l l^{-1} \in \mathcal{F}^{\perp}$, the extremalization of the action (32) leads to the result :

$$
\partial_{\tau} l l^{-1}+\alpha_{+}\left(\partial_{\sigma} l l^{-1}\right)+R \alpha_{+}\left(\partial_{\sigma} l l^{-1}\right)-\alpha_{-}\left(\partial_{\sigma} l l^{-1}\right)+R^{*} \alpha_{-}\left(\partial_{\sigma} l l^{-1}\right) \in \mathcal{F},
$$

or written in the light-cone coordinates:

$$
\alpha_{+}\left(\partial_{+} l l^{-1}\right)+R \alpha_{+}\left(\partial_{+} l l^{-1}\right)+\alpha_{-}\left(\partial_{-} l l^{-1}\right)-R^{*} \alpha_{-}\left(\partial_{-} l l^{-1}\right) \in \mathcal{F} .
$$

The second order action: In this paragraph we will present an algebraic derivation of the dual pair of the $\sigma$ models from the first order action (32). As before, since the double $D$ is perfect, we can represent any field $l(\sigma, \tau) \in D$ as $l(\sigma, \tau)=g(\sigma, \tau) \hat{h}(\sigma, \tau)$ with $g(\sigma, \tau) \in G$ and $\hat{h}(\sigma, \tau) \in \hat{G}$. With this parametrization, we can write the action (32) as:

$$
\mathcal{S}(g, \hat{h}, \Lambda)=\int\left\langle\partial_{\tau} g g^{-1} \mid \partial_{\sigma} l l^{-1}\right\rangle+\left\langle\partial_{\sigma} l l^{-1} \mid \Lambda\right\rangle+\frac{1}{2} K\left(\partial_{\sigma} l l^{-1}\right) .
$$

The constraint is solved by restricting the variable $\partial_{\sigma} l l^{-1}$ on $\mathcal{F}^{\perp}$, which, following the Theorem 2, permits to perform the decomposition $\partial_{\sigma} l l^{-1}=$ $\alpha_{+}+\alpha_{-}+R \alpha_{+}-R^{*} \alpha_{-}$. The action then becomes simply:

$$
\mathcal{S}(g, \hat{h})=\int\left\langle\partial_{\tau} g g^{-1} \mid \alpha_{+}+\alpha_{-}\right\rangle+\left\langle\alpha_{+} \mid R \alpha_{+}\right\rangle+\left\langle\alpha_{-} \mid R \alpha_{-}\right\rangle
$$

The elements $\alpha_{ \pm}$of $\hat{\mathcal{G}}$ are still related by the relation (15) as it can be seen by exactly the same reasoning as in Section 2:

$$
\left(R+\Pi^{g}\right) \alpha_{+}-\left(R+\Pi^{g}\right)^{*} \alpha_{-}=\partial_{\sigma} g g^{-1} .
$$

And from the first order action (32), we derive the same action as Eq.(17) with $M^{g}=\Pi^{g}$ directly:

$$
S(g, \hat{h})=-\frac{1}{2} \int\left\langle Y \mid\left(R+\Pi^{g}\right) Y\right\rangle+\left\langle Z-\partial_{\tau} g g^{-1}\right|\left(R+\Pi^{g}\right)^{-1}\left(Z+\partial_{\tau} g g^{-1}\right\rangle,
$$

where

$$
\begin{aligned}
Y & =2 \alpha_{+}-\left(R+\Pi^{g}\right)^{-1} \partial_{+} g g^{-1} \\
Z & =\left(R+\Pi^{g}\right) \alpha_{+}-\left(R+\Pi^{g}\right)^{*} \alpha_{-}=\partial_{\sigma} g g^{-1}
\end{aligned}
$$

The action (42) does not depend of the choice of the $\alpha_{ \pm}$operators. Indeed, if we make the transformation $\alpha_{ \pm} \rightarrow \alpha_{ \pm} \pm \Phi$ with $\Phi \in \operatorname{Ker}\left(R+R^{*}\right)$, the action (42) remains invariant. To see it, in a first time we observe that the 
quantity $Z$ is unchanged under that transformation. Secondly, under the same transformation the quantity $Y$ becomes $Y+2 \Phi$, and the quadratic form $\left\langle Y \mid\left(R+\Pi^{g}\right) Y\right\rangle$ becomes:

$$
\begin{aligned}
\left\langle Y+2 \Phi \mid\left(R+\Pi^{g}\right)(Y+2 \Phi)\right\rangle & =\frac{1}{2}\left\langle Y+2 \Phi \mid\left(R+R^{*}\right)(Y+2 \Phi)\right\rangle \\
& =\frac{1}{2}\left\langle Y \mid\left(R+R^{*}\right) Y\right\rangle \\
& =\left\langle Y \mid\left(R+\Pi^{g}\right) Y\right\rangle .
\end{aligned}
$$

The quadratic form $\left\langle Y \mid\left(R+\Pi^{g}\right) Y\right\rangle$ is therefore naturally defined on the coset $\hat{\mathcal{G}} / \operatorname{Ker}\left(R+R^{*}\right)$, which makes possible to write (42) as:

$$
S(g, \hat{h})=-\frac{1}{2} \int \frac{1}{2}\left\langle\pi(Y) \mid\left(R+R^{*}\right)_{\pi} \pi(Y)\right\rangle-\left\langle\partial_{-} g g^{-1} \mid\left(R+\Pi^{g}\right)^{-1} \partial_{+} g g^{-1}\right\rangle,
$$

where $\pi$ is the canonical projection $\pi: \hat{\mathcal{G}} \rightarrow \hat{\mathcal{G}} / \operatorname{Ker}\left(R+R^{*}\right)$, and the operator $\left(R+R^{*}\right)_{\pi}$ is nothing but the operator $\left(R+R^{*}\right)$ acting on the coset $\hat{\mathcal{G}} /$ $\operatorname{Ker}\left(R+R^{*}\right)$.

The following Lemma 1 shows that varying $\hat{h}$ in $l=g \hat{h}$ while keeping fixed $g$ and respecting the constraint $\partial_{\sigma} l l^{-1} \in \mathcal{F}^{\perp}$ is the same thing as varying $\pi(Y)$. This implies that we obtain from (46) directly the second order action:

$$
S(g)=\frac{1}{2} \int\left\langle\partial_{-} g g^{-1} \mid\left(R+\Pi^{g}\right)^{-1} \partial_{+} g g^{-1}\right\rangle, \quad g \in G .
$$

Indeed in (46), there is no linear term in $\pi(Y)$, therefore varying with respect to $\pi(Y)$ amounts simply to the suppression of the quadratic term in $\pi(Y)$.

Lemma 1. It holds that:

$$
\pi(Y)=2\left(R+R^{*}\right)_{\pi}^{-1}\left(\left(R+\Pi^{g}\right)^{*} \hat{J} \operatorname{Ad}_{g} \hat{J} \partial_{\sigma} \hat{h} \hat{h}^{-1}+\partial_{\sigma} g g^{-1}\right)-\pi\left(\left(R+\Pi^{g}\right)^{-1} \partial_{+} g g^{-1}\right) .
$$

Proof. Firstly, we have to show that the formula (48) is well defined, which means that the quantity $V=\left(R+\Pi^{g}\right)^{*} \hat{J} \operatorname{Ad}_{g} \hat{J} \partial_{\sigma} \hat{h} \hat{h}^{-1}+\partial_{\sigma} g g^{-1}$ is in the image of $R+R^{*}$ whereas $\partial_{\sigma} l l^{-1} \in \mathcal{F}^{\perp}$.

Let us consider any $\partial_{\sigma} l l^{-1} \in \mathcal{F}^{\perp}$, which means that for all $\gamma \in \operatorname{Ker}\left(R+R^{*}\right)$ :

$$
\left\langle\operatorname{Ad}_{g} \partial_{\sigma} \hat{h} \hat{h}^{-1}+\partial_{\sigma} g g^{-1} \mid \gamma+R \gamma\right\rangle=0,
$$

hence

$$
\left\langle\left(R+\Pi^{g}\right)^{*} \hat{J} \operatorname{Ad}_{g} \hat{J} \partial_{\sigma} \hat{h} \hat{h}^{-1}+\partial_{\sigma} g g^{-1} \mid \gamma\right\rangle=\langle V \mid \gamma\rangle=0 .
$$


Therefore $V \in \operatorname{Ker}\left(R+R^{*}\right)^{0} \subset \mathcal{G}$, it remains to prove that $\operatorname{Im}\left(R+R^{*}\right)=$ $\operatorname{Ker}\left(R+R^{*}\right)^{0}$.

We can immediately see that $\operatorname{Im}\left(R+R^{*}\right) \subset \operatorname{Ker}\left(R+R^{*}\right)^{0}$, indeed for $\gamma \in$ $\operatorname{Ker}\left(R+R^{*}\right)$ we have $\left\langle\gamma \mid\left(R+R^{*}\right) X\right\rangle=\left\langle\left(R+R^{*}\right) \gamma \mid X\right\rangle=0$, where $X \in \hat{\mathcal{G}}$. Moreover, since the bilinear form $\langle. \mid$.$\rangle is non degenerate we have the relation$ $\operatorname{dim} \operatorname{Ker}\left(R+R^{*}\right)^{0}=n-\operatorname{dim} \operatorname{Ker}\left(R+R^{*}\right)$. From the rank-nullity theorem, we obtain that $\operatorname{dim} \operatorname{Ker}\left(R+R^{*}\right)+\operatorname{dim} \operatorname{Im}\left(R+R^{*}\right)=n$, together with the previous relation we conclude that $\operatorname{dim} \operatorname{Ker}\left(R+R^{*}\right)^{0}=\operatorname{dim} \operatorname{Im}\left(R+R^{*}\right)$ and $\operatorname{Im}\left(R+R^{*}\right)=\operatorname{Ker}\left(R+R^{*}\right)^{0}$. Consequently, $V \in \operatorname{Im}\left(R+R^{*}\right)$.

Secondly, let us prove the relation (48). From the decomposition of $\partial_{\sigma} l l^{-1} \in$ $\mathcal{F}^{\perp}$ in the Theorem 2 , we obtain:

$$
\left(R+R^{*}\right) \alpha_{+}=\left(R^{*} \hat{J} \operatorname{Ad}_{g} \hat{J}+J \operatorname{Ad}_{g} \hat{J}\right) \partial_{\sigma} \hat{h} \hat{h}^{-1}+\partial_{\sigma} g g^{-1}=V .
$$

The relation (51) can be expressed in term of the quantity $Y$ (cf Eq.(43)):

$$
\left(R+R^{*}\right) Y=2 V-\left(R+R^{*}\right)\left(R+\Pi^{g}\right)^{-1} \partial_{+} g g^{-1} .
$$

Hence:

$$
\left(R+R^{*}\right)_{\pi} \pi(Y)=2 V-\left(R+R^{*}\right)_{\pi} \pi\left(\left(R+\Pi^{g}\right)^{-1} \partial_{+} g g^{-1}\right) .
$$

Since $\left(R+R^{*}\right)_{\pi}$ is an invertible operator on $\hat{\mathcal{G}} / \operatorname{Ker}\left(R+R^{*}\right)$, by inverting (53) we obtain the desired result:

$$
\pi(Y)=2\left(R+R^{*}\right)_{\pi}^{-1} V-\pi\left(\left(R+\Pi^{g}\right)^{-1} \partial_{+} g g^{-1}\right) .
$$

Remark: If we denote $p$ the dimension of $\operatorname{Ker}\left(R+R^{*}\right)$, then the $n-p$ variables contained in $\pi(Y)$ are related by the invertible relation (48) to the $n-p$ independent components of $\partial_{\sigma} \hat{h} \hat{h}^{-1}$ (Note that there are only $n-p$ independant components in $\partial_{\sigma} \hat{h} \hat{h}^{-1}$, because $p$ components of $\partial_{\sigma} \hat{h} \hat{h}^{-1}$ are determined by the relation $\partial_{\sigma} l l^{-1} \in \mathcal{F}^{\perp}$ ).

The dual second order action with respect to the action (47) is still obtain by taking the dual ansatz $l(\sigma, \tau)=\hat{g}(\sigma, \tau) h(\sigma, \tau) \in D$ with $\hat{g}(\sigma, \tau) \in \hat{G}$ and $h(\sigma, \tau) \in G$, and the well-known relation for the $\hat{R}: \mathcal{G} \rightarrow \hat{\mathcal{G}}$ operator:

$$
\hat{R}=R^{-1} \text {. }
$$

The same reasoning leads to the dual second order action on $\hat{G}$ :

$$
S(\hat{g})=\frac{1}{2} \int\left\langle\partial_{-} \hat{g} \hat{g}^{-1} \mid\left(R^{-1}+\hat{\Pi}^{\hat{g}}\right)^{-1} \partial_{+} \hat{g} \hat{g}^{-1}\right\rangle, \quad \hat{g} \in \hat{G} .
$$


The standard dressing cosets: Suppose that the linear subspace $\mathcal{F}$ is a Lie subalgebra of $\mathcal{D}$, whose Lie group $F$ is a connected Lie subgroup of $D$. Then:

Theorem 3. The first order action (32) develops a gauge symmetry with $F$ as the gauge group if the operator $R$ verifies:

$$
J \operatorname{Ad}_{f} \hat{J}+J\left[\operatorname{Ad}_{f}, R\right] \hat{J}=R \operatorname{Ad}_{f} R, \forall f \in F .
$$

For $f(\sigma, \tau) \in F$, the revelant gauge transformations read:

$l(\sigma, \tau) \rightarrow f(\sigma, \tau) l(\sigma, \tau), \quad \Lambda(\sigma, \tau) \rightarrow f(\sigma, \tau) \Lambda(\sigma, \tau) f(\sigma, \tau)^{-1}-\partial_{\tau} f(\sigma, \tau) f(\sigma, \tau)^{-1}$

Remark: The condition (57) is equivalent to the stability of the subspaces $\mathcal{R}$ and $\mathcal{R}^{\perp}$ by the adjoint action of $F$ as required in [2]. This fact will show up in the proof of the Theorem 3. Furthermore, in Section 5, we will show that the previous hypothesis (isotropy of $\mathcal{F}$ and the condition (57)) responsible for the gauge invariance of the first order action (32), will imply also a gauge invariance for the second order actions (47) and (56).

Proof. We use the well-known Polyakov-Wiegmann formula [7]:

$$
\mathcal{S}_{0}(f l)=\mathcal{S}_{0}(l)+\mathcal{S}_{0}(f)+\int\left\langle f^{-1} \partial_{\tau} f \mid \partial_{\sigma} l l^{-1}\right\rangle,
$$

where

$$
\mathcal{S}_{0}(l)=\frac{1}{2} \int\left\langle\partial_{\tau} l l^{-1} \mid \partial_{\sigma} l l^{-1}\right\rangle+\frac{1}{6} d^{-1}\left\langle d l l^{-1} \wedge\left[d l l^{-1} \wedge d l l^{-1}\right]\right\rangle,
$$

and the formula

$$
\partial_{\sigma} l l^{-1} \rightarrow \partial_{\sigma} f f^{-1}+\mathrm{Ad}_{f} \partial_{\sigma} l l^{-1} .
$$

The isotropy of $\mathcal{F}$ gives immediatly $\mathcal{S}_{0}(f)=0$, and with the constraint $\partial_{\sigma} l l^{-1} \in \mathcal{F}^{\perp}$ both ensure the gauge invariance of $\mathcal{S}_{0}(l)$.

Concerning gauge invariance of the term containing the quadratic form $K$ in the action (32), we can show that the transformation (60) leads to the following expression $K\left(\partial_{\sigma} l l^{-1}\right) \rightarrow K\left(\partial_{\sigma} f f^{-1}+\mathrm{Ad}_{f} \partial_{\sigma} l l^{-1}\right)$. By remarking that $\alpha_{ \pm}\left(\partial_{\sigma} f f^{-1}\right) \in \operatorname{Ker}\left(R+R^{*}\right)$, the expression of the quadratic form becomes simply $K\left(\partial_{\sigma} f f^{-1}+\operatorname{Ad}_{f} \partial_{\sigma} l l^{-1}\right)=K\left(\operatorname{Ad}_{f} \partial_{\sigma} l l^{-1}\right)$. We will prove that $K\left(\operatorname{Ad}_{f} \partial_{\sigma} l l^{-1}\right)=K\left(\partial_{\sigma} l l^{-1}\right)$ in several steps.

Firstly, we will show that taking $X \in \mathcal{F}^{\perp}=\mathcal{R}+\mathcal{R}^{\perp}$ then $\operatorname{Ad}_{f} \mathcal{F}^{\perp} \subset \mathcal{F}^{\perp}$. Since $X \in \mathcal{F}^{\perp}$ it can be decomposed by using the relation (33) as $X=$ 
$\alpha+R \alpha+\beta-R^{*} \beta$ with $(\alpha, \beta) \in \hat{\mathcal{G}}$. Thus if we act on $X \in \mathcal{R}+\mathcal{R}^{\perp}$ by the adjoint action of any element $f \in F$, we obtain on $\mathcal{G}$ and $\hat{\mathcal{G}}$ :

$$
\begin{aligned}
& \delta \equiv \operatorname{JAd}_{f} \hat{J} \alpha+J \operatorname{Ad}_{f} J R \alpha \in \mathcal{G} \\
& \hat{\delta} \equiv \hat{J} \operatorname{Ad}_{f} \hat{J} \alpha+\hat{J} \operatorname{Ad}_{f} J R \alpha \in \hat{\mathcal{G}} .
\end{aligned}
$$

Moreover, the relation (62) can be modified by using the condition (57) on $R$ as:

$$
\begin{aligned}
\hat{J} \operatorname{Ad}_{f} \hat{J} \alpha+\hat{J} \operatorname{Ad}_{f} J R \alpha & =R \hat{J} \operatorname{Ad}_{f} \hat{J} \alpha+R \hat{J} \operatorname{Ad}_{f} J R \alpha \\
& =R \hat{\delta} .
\end{aligned}
$$

It implies that $\operatorname{Ad}_{f} \mathcal{R} \subset \mathcal{R}$. The same reasoning holds with the quantity $\beta$ which leads to $\operatorname{Ad}_{f} \mathcal{R}^{\perp} \subset \mathcal{R}^{\perp}$, and consequently $\operatorname{Ad}_{f} \mathcal{F}^{\perp} \subset \mathcal{F}^{\perp}$.

Secondly, since $\operatorname{Ad}_{f} X \in \mathcal{F}^{\perp}$ for $X \in \mathcal{F}^{\perp}$, we can use now the relation (33) to decompose it as:

$$
\begin{aligned}
\operatorname{Ad}_{f} X & =\operatorname{Ad}_{f} \alpha_{+}(X)+\operatorname{Ad}_{f} \alpha_{-}(X)+\operatorname{Ad}_{f} R \alpha_{+}(X)-\operatorname{Ad}_{f} R^{*} \alpha_{-}(X) \\
\operatorname{Ad}_{f} X & =\alpha_{+}\left(\operatorname{Ad}_{f} X\right)+\alpha_{-}\left(\operatorname{Ad}_{f} X\right)+R \alpha_{+}\left(\operatorname{Ad}_{f} X\right)-R^{*} \alpha_{-}\left(\operatorname{Ad}_{f} X\right) .
\end{aligned}
$$

Then, on the subspace $\mathcal{R}$ the equality between the previous relations leads to:

$$
\begin{aligned}
J \operatorname{Ad}_{f} \alpha_{+}(X)+J \operatorname{Ad}_{f} R \alpha_{+}(X) & =R \alpha_{+}\left(\operatorname{Ad}_{f} X\right) \\
\hat{J} \operatorname{Ad}_{f} \alpha_{+}(X)+\hat{J} \operatorname{Ad}_{f} R \alpha_{+}(X) & =\alpha_{+}\left(\operatorname{Ad}_{f} X\right) .
\end{aligned}
$$

Thirdly, let us take for example the first term of $K\left(\operatorname{Ad}_{f} X\right)$, and use the relations (67) and (68) to write:

$$
\begin{aligned}
\left\langle\alpha_{+}\left(\operatorname{Ad}_{f} X\right) \mid R \alpha_{+}\left(\operatorname{Ad}_{f} X\right)\right\rangle= & \left\langle R \alpha_{+}(X) \mid \alpha_{+}(X)\right\rangle+\left\langle\Xi_{1}^{f} R \alpha_{+}(X) \mid R \alpha_{+}(X)\right\rangle \\
& +\left\langle\Xi_{2}^{f} \alpha_{+}(X) \mid \alpha_{+}(X)\right\rangle .
\end{aligned}
$$

Since $\Xi_{1}^{f}=\hat{J} \operatorname{Ad}_{f^{-1}} \hat{J} \operatorname{Ad}_{f} J$ and $\Xi_{2}^{f}=J \operatorname{Ad}_{f^{-1}} \hat{J} \operatorname{Ad}_{f} \hat{J}$ are two skew-symetric operators in a symetric form, thus the last two terms are null. The case $\left\langle\alpha_{-}\left(\operatorname{Ad}_{f} X\right) \mid R \alpha_{-}\left(\operatorname{Ad}_{f} X\right)\right\rangle$ can be done in a similar way.

\section{Models with $\operatorname{Ker} R=\operatorname{Ker} R^{*} \neq\{0\}$ and $\operatorname{Ker}(R+$ $\left.R^{*}\right) \neq\{0\}$}

In Section 3, we demanded from the operators $R$ and $\hat{R}$ to have the trivial kernel; it is this last constraint which will be relaxed in that current section. We will see that, under some hypothesis on the operators $R$ and $\hat{R}$, the duality pair of non linear $\sigma$-models can be established even in this more general case. 
Preliminaries: We start with two operators $R: \operatorname{Dom}(R) \rightarrow \operatorname{Im}(R)$ and $\hat{R}: \operatorname{Dom}(\hat{R}) \rightarrow \operatorname{Im}(\hat{R})$, where $\operatorname{Dom} R$ and $\operatorname{Im}(\hat{R})$ are subsets of $\hat{\mathcal{G}}$, and $\operatorname{Dom} \hat{R}$ and $\operatorname{Im}(R)$ are subsets of $\mathcal{G}$. Those operators verify the following hypothesis:

1. $\operatorname{Ker} R=\operatorname{Ker} R^{*}, \operatorname{Ker} \hat{R}=\operatorname{Ker} \hat{R}^{*}$

2. $\left(\left.R\right|_{\operatorname{Im} \hat{R}}\right)^{-1}=\left.\hat{R}\right|_{\operatorname{Im} R}$

3. $(\operatorname{Ker} \hat{R})^{0}=\operatorname{Dom} R$ and $(\operatorname{Ker} R)^{0}=\operatorname{Dom} \hat{R}$

4. $\operatorname{Im} \hat{R} \subset \operatorname{Dom} R$ and $\operatorname{Im} R \subset \operatorname{Dom} \hat{R}$

5. $\operatorname{Dom} R=\operatorname{Dom} R^{*}, \operatorname{Dom} \hat{R}=\operatorname{Dom} \hat{R}^{*}$

6. The bilinear form $\langle. \mid$.$\rangle restricted to \operatorname{Im} R \oplus \operatorname{Im} \hat{R}$ is non degenerate

The first hypothesis is exactly the same as previously, the second one ensures the duality, the third one ensures the orthogonality of certain subspaces $\mathcal{R}$ and $\mathcal{R}^{\perp}$ of $\mathcal{D}$ that will generalize the subspaces $\mathcal{R}$ and $\mathcal{R}^{\perp}$ considered in Section 2 and 3, and the importance of the fifth shows up in the definition of the quadratic form $K$.

Remarks: Firstly, the requirements 1-4 imply the following decompositions for the subspaces $\operatorname{Dom} R$ and $\operatorname{Dom} \hat{R}$ :

$$
\operatorname{Dom} R=\operatorname{Im} \hat{R} \oplus \operatorname{Ker} R, \quad \operatorname{Dom} \hat{R}=\operatorname{Im} R \oplus \operatorname{Ker} \hat{R} .
$$

Secondly, the relation (28) between the kernels of $R+R^{*}$ and $\hat{R}+\hat{R}^{*}$ is changed, indeed both operators $R$ and $\hat{R}$ have non trivial kernels, with the result that the link between the kernels becomes:

$$
\left.\operatorname{Ker}\left(\hat{R}+\hat{R}^{*}\right)\right|_{\operatorname{Im} R}=\left.\left.R\right|_{\operatorname{Im} \hat{R}} \operatorname{Ker}\left(R+R^{*}\right)\right|_{\operatorname{Im} \hat{R}}
$$

In our new case, the linear subspaces $\mathcal{R}, \mathcal{R}^{\perp}$ and $\mathcal{F}$ are defined as follows:

$$
\begin{aligned}
\mathcal{R} & =\operatorname{Ker} \hat{R} \oplus\{\gamma+R \gamma, \gamma \in \operatorname{Dom} R\} \\
\mathcal{R}^{\perp} & =\operatorname{Ker} \hat{R} \oplus\left\{\gamma-R^{*} \gamma, \gamma \in \operatorname{Dom} R^{*}\right\} \\
\mathcal{F}=\mathcal{R} \cap \mathcal{R}^{\perp} & =\operatorname{Ker} \hat{R} \oplus\left\{\gamma+R \gamma, \gamma \in \operatorname{Ker}\left(R+R^{*}\right)\right\} \\
& =\operatorname{Ker} \hat{R} \oplus\left\{\gamma-R^{*} \gamma, \gamma \in \operatorname{Ker}\left(R+R^{*}\right)\right\}
\end{aligned}
$$


Remark: The subspaces $\mathcal{R}$ and $\mathcal{R}^{\perp}$ can be rewritten in a duality invariant way:

$$
\begin{aligned}
\mathcal{R} & =\operatorname{Ker} \hat{R} \oplus \operatorname{Ker} R \oplus\{\gamma+R \gamma, \gamma \in \operatorname{Im} \hat{R}\} \\
& =\operatorname{Ker} \hat{R} \oplus \operatorname{Ker} R \oplus\{\tilde{\gamma}+\hat{R} \tilde{\gamma}, \tilde{\gamma} \in \operatorname{Im} R\} \\
\mathcal{R}^{\perp} & =\operatorname{Ker} \hat{R} \oplus \operatorname{Ker} R \oplus\left\{\gamma-R^{*} \gamma, \gamma \in \operatorname{Im} \hat{R}\right\} \\
& =\operatorname{Ker} \hat{R} \oplus \operatorname{Ker} R \oplus\left\{\tilde{\gamma}-\hat{R}^{*} \tilde{\gamma}, \tilde{\gamma} \in \operatorname{Im} R\right\} \\
\mathcal{F} & =\operatorname{Ker} \hat{R} \oplus \operatorname{Ker} R \oplus\left\{\gamma+R \gamma, \gamma \in \operatorname{Im} \hat{R} \cap \operatorname{Ker}\left(R+R^{*}\right)\right\} \\
& =\operatorname{Ker} \hat{R} \oplus \operatorname{Ker} R \oplus\left\{\tilde{\gamma}+\hat{R} \tilde{\gamma}, \tilde{\gamma} \in \operatorname{Im} R \cap \operatorname{Ker}\left(\hat{R}+\hat{R}^{*}\right)\right\}
\end{aligned}
$$

First order action: The expression of the first order action is exactly the same as in Eq.(32), only the properties of the $R$ and $\hat{R}$ change. We still consider a $D$-valued field $l(\sigma, \tau)$ and an $\mathcal{F}$-valued field $\Lambda(\sigma, \tau)$, and we write the first order action as:

$\mathcal{S}(l)=\frac{1}{2} \int\left\langle\partial_{\tau} l l^{-1} \mid \partial_{\sigma} l l^{-1}\right\rangle+\frac{1}{6} d^{-1}\left\langle d l l^{-1} \wedge\left[d l l^{-1} \wedge d l l^{-1}\right]\right\rangle+K\left(\partial_{\sigma} l l^{-1}\right)+\left\langle\Lambda \mid \partial_{\sigma} l l^{-1}\right\rangle$.

Again, the field $\Lambda$ is the Lagrange multiplier the variation of which imposes the constraint $\partial_{\sigma} l l^{-1} \in \mathcal{F}^{\perp}$, and in order to specify $K$ we need the following generalization of the Theorem 2 :

Theorem 4. There exists a pair of linear operators $\alpha_{ \pm}: \mathcal{F}^{\perp} \rightarrow \operatorname{Dom} R \subset \hat{\mathcal{G}}$ and an unique linear operator $\alpha_{0}: \mathcal{F}^{\perp} \rightarrow \operatorname{Ker} \hat{R}$ such that every $X \in \mathcal{F}^{\perp}$ can be decomposed as:

$$
X=\alpha_{+}(X)+\alpha_{-}(X)+R \alpha_{+}(X)-R^{*} \alpha_{-}(X)+\alpha_{0}(X) .
$$

Proof. The proof is similar to that of the Theorem 2, but adapted to the new definition of the $\mathcal{F}$ subspace.

We can easily verify that $\mathcal{R}+\mathcal{R}^{\perp} \subset \mathcal{F}^{\perp}$, indeed because of $\langle\operatorname{Ker} \hat{R} \mid \operatorname{Dom} R\rangle=0$ the two subspaces $\mathcal{R}$ and $\mathcal{R}^{\perp}$ remain orthogonal to $\mathcal{F}$. Let us calculate the dimension of $\mathcal{R}+\mathcal{R}^{\perp}$ :

$$
\operatorname{dim}\left(\mathcal{R}+\mathcal{R}^{\perp}\right)=2 \operatorname{dim}(\operatorname{Dom} R)+2 \operatorname{dim} \operatorname{Ker} \hat{R}-\operatorname{dim} \mathcal{F} .
$$

The dimension of $\operatorname{Dom} R$ can be found from the relation $\operatorname{Dom} R=(\operatorname{Ker} \hat{R})^{0}$, which gives:

$$
\operatorname{dim}(\operatorname{Dom} R)=n-\operatorname{dim}(\operatorname{Ker} \hat{R}),
$$

and implies:

$$
\operatorname{dim}\left(\mathcal{R}+\mathcal{R}^{\perp}\right)=2 n-\operatorname{dim} \mathcal{F} .
$$


Consider now two subspaces $\mathcal{A}$ and $\hat{\mathcal{A}}$ such that $\mathcal{G}=\operatorname{Dom} \hat{R} \oplus \hat{\mathcal{A}}$ and $\hat{\mathcal{G}}=$ $\operatorname{Dom} R \oplus \mathcal{A}$. Thus it implies that $\operatorname{dim} \mathcal{A}=\operatorname{dim} \operatorname{Ker} \hat{R}$ and $\operatorname{dim} \hat{\mathcal{A}}=\operatorname{dim} \operatorname{Ker} R$. Futhermore, we denote $\left.R\right|_{\operatorname{Im} \hat{R}}: \operatorname{Im} \hat{R} \rightarrow \operatorname{Im} R$ the restriction of $R$ on $\operatorname{Im} \hat{R}$. With this convention the linear subspace $\mathcal{F}$ can be written:

$$
\mathcal{F}=\operatorname{Ker} R \oplus \operatorname{Ker} \hat{R} \oplus\left\{\gamma+\left.R\right|_{\operatorname{Im} \hat{R}} \gamma,\left.\gamma \in \operatorname{Ker}\left(R+R^{*}\right)\right|_{\operatorname{Im} \hat{R}}\right\}
$$

We choose any complement subspace $\mathcal{U}$ of $\left.\operatorname{Ker}\left(R+R^{*}\right)\right|_{\operatorname{Im} \hat{R}} ^{0}$ in $\operatorname{Im} \hat{R}$, where $\left.\operatorname{Ker}\left(R+R^{*}\right)\right|_{\operatorname{Im} \hat{R}} ^{0}$ is the annihilator of $\left.\operatorname{Ker}\left(R+R^{*}\right)\right|_{\operatorname{Im} \hat{R}}$ in $\operatorname{Im} \hat{R}$. Then we can write $\operatorname{Im} \hat{R}=\left.\operatorname{Ker}\left(R+R^{*}\right)\right|_{\operatorname{Im} \hat{R}} ^{0} \oplus \mathcal{U}$, and $\left.\operatorname{Ker}\left(R+R^{*}\right)\right|_{\operatorname{Im} \hat{R}} ^{0} \cap \mathcal{U}=\{0\}$. With this definition we directly obtain the dimension of the subspace $\mathcal{U}$ :

$$
\operatorname{dim} \mathcal{U}=\left.\operatorname{dim} \operatorname{Ker}\left(R+R^{*}\right)\right|_{\operatorname{Im} \hat{R}}
$$

We consider the following subspace $(\mathcal{A} \oplus \hat{\mathcal{A}})+\mathcal{U}+\mathcal{F}^{\perp}$, and we shall prove that all the sums are direct.

We want to show that $\mathcal{A} \cap \mathcal{F}^{\perp}=\{0\}$. Suppose that there exists a non vanishing $a \in \mathcal{A}$ such that $\langle a \mid \hat{k}\rangle=0$ for all $\hat{k} \in \operatorname{Ker} \hat{R} \subset \mathcal{F}$, then $a \in(\operatorname{Ker} \hat{R})^{0}=$ $\operatorname{Dom} R$. Since $\mathcal{A} \cap \operatorname{Dom} R=\{0\}$, it implies that $a=0$ in contradiction with the hypothesis and we obtain that $\mathcal{A} \cap \mathcal{F}^{\perp}=\{0\}$. The same reasoning holds for the subspace $\hat{A}$, leading to $\hat{A} \cap \mathcal{F}^{\perp}=\{0\}$.

Let us show now that $\mathcal{U} \cap \mathcal{F}^{\perp}=\{0\}$. Suppose that there exists a non vanishing $u \in \mathcal{U}$ such that $\langle u \mid \gamma\rangle=0$ for all $\left.\gamma \in \operatorname{Ker}\left(R+R^{*}\right)\right|_{\operatorname{Im} \hat{R}}$, then $\left.u \in \operatorname{Ker}\left(R+R^{*}\right)\right|_{\operatorname{Im} \hat{R}} ^{0}$. But $\left.\mathcal{U} \cap \operatorname{Ker}\left(R+R^{*}\right)\right|_{\operatorname{Im} \hat{R}} ^{0}=\{0\}$, it implies that $u=0$ contradicting the hypothesis. We obtain $\mathcal{U} \cap \mathcal{F}^{\perp}=\{0\}$.

Moreover $\mathcal{U} \cap(A \oplus \hat{A})=\{0\}$, because $\mathcal{U} \subset \operatorname{Im} \hat{R}$ and $\operatorname{Im} \hat{R} \cap A=\operatorname{Im} \hat{R} \cap \hat{A}=\{0\}$. We can conclude that the following sum $A \oplus \hat{A} \oplus \mathcal{U} \oplus \mathcal{F}^{\perp} \subset \mathcal{D}$ is direct. Now observe that $\operatorname{dim}(A \oplus \hat{A} \oplus \mathcal{U})=\operatorname{dim} \mathcal{F}$, since we have already shown that $\operatorname{dim} \mathcal{U}=\left.\operatorname{dim} \operatorname{Ker}\left(R+R^{*}\right)\right|_{\operatorname{Im} \hat{R}}, \operatorname{dim} \mathcal{A}=\operatorname{dim} \operatorname{Ker} \hat{R}$ and $\operatorname{dim} \hat{\mathcal{A}}=\operatorname{dim} \operatorname{Ker} R$. From the fact that $A \oplus \hat{A} \oplus \mathcal{U} \oplus \mathcal{F}^{\perp} \subset \mathcal{D}$, we obtain the relation for the dimensions: $\operatorname{dim} \mathcal{F}^{\perp}+\operatorname{dim} \mathcal{F} \leq 2 n$.

From relation (84) we obtained $2 n=\operatorname{dim} \mathcal{F}+\operatorname{dim}\left(\mathcal{R}+\mathcal{R}^{\perp}\right)$, moreover we know that $\mathcal{R}+\mathcal{R}^{\perp} \subset \mathcal{F}^{\perp}$. Thus from the inequality $\operatorname{dim} \mathcal{F}^{\perp}+\operatorname{dim} \mathcal{F} \leq 2 n$ we observe that $\operatorname{dim} \mathcal{F}^{\perp}=2 n-\operatorname{dim} \mathcal{F}=\operatorname{dim}\left(\mathcal{R}+\mathcal{R}^{\perp}\right)$, which implies that $\mathcal{R}+\mathcal{R}^{\perp}=\mathcal{F}^{\perp}$.

Thus, for any $X \in \mathcal{F}^{\perp}=\mathcal{R}+\mathcal{R}^{\perp}$ we can decompose it as:

$$
X=\alpha_{+}(X)+R \alpha_{+}(X)+\alpha_{-}(X)-R^{*} \alpha_{-}(X)+\alpha_{0}(X),
$$

with $\left(\alpha_{+}, \alpha_{-}\right) \in \operatorname{Dom} R, \alpha_{0} \in \operatorname{Ker} \hat{R}$.

We remark that the operator $\alpha_{0}$ is nothing but the identity on $\operatorname{Ker} \hat{R}$ and the null operator on the rest of $\mathcal{F}^{\perp}$. 
The quadratic form $K$ has the same expression as Eq.(366):

$$
K(X)=2\left\langle\alpha_{+}(X), R \alpha_{+}(X)\right\rangle+2\left\langle\alpha_{-}(X), R \alpha_{-}(X)\right\rangle, \forall X \in \mathcal{F}^{\perp},
$$

and $K$ is well defined on $\mathcal{F}^{\perp}$ since we required $\operatorname{Dom} R=\operatorname{Dom} R^{*}$.

The second order action: Since the double $D$ is perfect, we parametrize any field $l(\sigma, \tau) \in D$ as $l(\sigma, \tau)=g(\sigma, \tau) \hat{h}(\sigma, \tau)$ with $g(\sigma, \tau) \in G$ and $\hat{h}(\sigma, \tau) \in$ $\hat{G}$. We obtain the action:

$$
\mathcal{S}(g, \hat{h}, \Lambda)=\int\left\langle\partial_{\tau} g g^{-1} \mid \partial_{\sigma} l l^{-1}\right\rangle+\left\langle\partial_{\sigma} l l^{-1} \mid \Lambda\right\rangle+\frac{1}{2} K\left(\partial_{\sigma} l l^{-1}\right)
$$

and:

$$
\mathcal{S}(g, \hat{h}, \Lambda)=\int\left\langle\partial_{\tau} g g^{-1} \mid \alpha_{+}+\alpha_{-}\right\rangle+\left\langle\alpha_{+} \mid R \alpha_{+}\right\rangle+\left\langle\alpha_{-} \mid R \alpha_{-}\right\rangle,
$$

where we used in (90) the decomposition $\partial_{\sigma} l l^{-1}=\alpha_{+}+\alpha_{-}+R \alpha_{+}-R^{*} \alpha_{-}+\alpha_{0}$ on $\mathcal{F}^{\perp}$.

Furthermore, from the decomposition (82) considered for $X=\partial_{\sigma} l l^{-1}=$ $\partial_{\sigma} g g^{-1}+\operatorname{Ad}_{g} \partial_{\sigma} \hat{h} \hat{h}^{-1}$, we obtain:

$$
\partial_{\sigma} g g^{-1}=\left(R+\Pi^{g}\right) \alpha_{+}-\left(R+\Pi^{g}\right) \alpha_{-}+\alpha_{0} .
$$

We can apply to the previous relation a projector $\rho$ from $\mathcal{G}$ to $\operatorname{Im} R \oplus \hat{\mathcal{A}}$ and such that $\operatorname{Ker} \rho=\operatorname{Ker} \hat{R}$, then the relation (91) becomes:

$$
\rho\left(\partial g g^{-1}\right)=\left(R+\rho \circ \Pi^{g}\right) \alpha_{+}-\left(R+\rho \circ \Pi^{g}\right) \alpha_{-},
$$

with the operator $\left(R+\rho \circ \Pi^{g}\right): \operatorname{Dom} R \rightarrow \operatorname{Im} R \oplus \hat{\mathcal{A}}$. Moreover, since $\langle\operatorname{Dom} R \mid \operatorname{Ker} \hat{R}\rangle=0$, the action (90) can be rewritten:

$$
\mathcal{S}(g, \hat{h}, \Lambda)=\int\left\langle\rho\left(\partial_{\tau} g g^{-1}\right) \mid \alpha_{+}+\alpha_{-}\right\rangle+\left\langle\alpha_{+} \mid R \alpha_{+}\right\rangle+\left\langle\alpha_{-} \mid R \alpha_{-}\right\rangle .
$$

And, with the following relations,

$$
\begin{aligned}
& \tilde{Y}=2 \alpha_{+}-\left(R+\rho \circ \Pi^{g}\right)^{-1} \rho\left(\partial_{+} g g^{-1}\right) \\
& \tilde{Z}=\left(R+\rho \circ \Pi^{g}\right) \alpha_{+}-\left(R+\rho \circ \Pi^{g}\right)^{*} \alpha_{-}=\rho\left(\partial_{\sigma} g g^{-1}\right)
\end{aligned}
$$

the action (93) can be written:

$$
\begin{aligned}
S(g, \hat{h}) & =-\frac{1}{2} \int\left\langle\tilde{Y} \mid\left(R+\rho \circ \Pi^{g}\right) \tilde{Y}\right\rangle \\
& +\left\langle\tilde{Z}-\rho\left(\partial_{\tau} g g^{-1}\right)\right|\left(R+\rho \circ \Pi^{g}\right)^{-1}\left(\tilde{Z}+\rho\left(\partial_{\tau} g g^{-1}\right)\right\rangle .
\end{aligned}
$$


Contrary to the previous case $(\operatorname{Ker} \hat{R}=\{0\})$, this time the variable $\tilde{Y}$ is defined on the $\operatorname{Dom} R \subset \hat{\mathcal{G}}$ and the variable $\tilde{Z}$ is defined on the subspace $\operatorname{Im} R \oplus \hat{\mathcal{A}}$ rather than on the whole $\mathcal{G}$ space.

The action (94) has exactly the same structure as the action (42) and leads, after the minimization, to the following second order action:

$$
\mathcal{S}(g)=\frac{1}{2} \int d \xi^{+} d \xi^{-}\left\langle\rho\left(\partial_{-} g g^{-1}\right) \mid\left(R+\rho \circ \Pi^{g}\right)^{-1} \rho\left(\partial_{+} g g^{-1}\right)\right\rangle, \quad g \in G .
$$

We won't do the calculus for the dual model, which is precisely the same as for the model on $G$, and leads to the following dual action:

$$
\mathcal{S}(\hat{g})=\frac{1}{2} \int d \xi^{+} d \xi^{-}\left\langle\tilde{\rho}\left(\partial_{-} \hat{g} \hat{g}^{-1}\right) \mid\left(\hat{R}+\tilde{\rho} \circ \hat{\Pi}^{\hat{g}}\right)^{-1} \tilde{\rho}\left(\partial_{+} \hat{g} \hat{g}^{-1}\right)\right\rangle, \quad \hat{g} \in \hat{G},
$$

where $\tilde{\rho}$ is the projector from $\hat{\mathcal{G}}$ to the subspace $\operatorname{Im} \hat{R} \oplus \mathcal{A}$ with $\operatorname{Ker} \hat{\rho}=\operatorname{Ker} R$.

Remarks: The duality relation $\hat{R}=R^{-1}$ of Section 2 and 3 has its analogue in the present section, indeed the operators $R$ and $\hat{R}$ are invertible on the respective subspaces $\operatorname{Im} \hat{R}$ and $\operatorname{Im} R$ and it holds $\left(\left.R\right|_{\operatorname{Im} \hat{R}}\right)^{-1}=\left.\hat{R}\right|_{\operatorname{Im} R}$.

The generalized dressing cosets : We suppose again that the linear subspace $\mathcal{F}$ is a Lie subalgebra of $\mathcal{D}$, whose Lie group $F$ is a connected Lie subgroup of $D$. Then:

Theorem 5. The first order action (81) develops a gauge symmetriy with $F$ as the gauge group if both operators $R$ and $\hat{R}$ verify on their domains:

$$
\begin{aligned}
J_{A d_{f}} \hat{J}+J\left[\operatorname{Ad}_{f}, R\right] \hat{J} & =R \operatorname{Ad}_{f} R, \\
\hat{J} \operatorname{Ad}_{f} J+\hat{J}\left[\operatorname{Ad}_{f}, \hat{R}\right] J & =\hat{R} \operatorname{Ad}_{f} \hat{R}, \forall f \in F .
\end{aligned}
$$

Remark: Before the proof, let us make a short remark; because of the relation

$$
\left(\left.R\right|_{\operatorname{Im} \hat{R}}\right)^{-1}=\left.\hat{R}\right|_{\operatorname{Im} R}
$$

the relations (97), (98) are equivalent on the subspaces $\operatorname{Im} \hat{R}$ and $\operatorname{Im} R$ respectively. However, since $\operatorname{Dom} R=\operatorname{Im} \hat{R} \oplus \operatorname{Ker} R$ and $\operatorname{Dom} \hat{R}=\operatorname{Im} R \oplus \operatorname{Ker} \hat{R}$, the relations (97), (98) give new conditions for $R$ and $\hat{R}$ on the kernels. If we choose $\delta \in \operatorname{Ker} \hat{R}$ and $\tilde{\delta} \in \operatorname{Ker} R$, then the relations (97), (98) give:

$$
\begin{aligned}
\hat{J} \operatorname{Ad}_{f} J \delta & =R \hat{J} \operatorname{Ad}_{f} J \tilde{\delta} \\
J_{A_{f}} \hat{J} \tilde{\delta} & =\hat{R} J \operatorname{Ad}_{f} \hat{J} \delta
\end{aligned}
$$

implying that $\operatorname{Ad}_{f} \operatorname{Ker} \hat{R} \subset \operatorname{Ker} \hat{R}$ and $\operatorname{Ad}_{f} \operatorname{Ker} R \subset \operatorname{Ker} R$. 
Proof. The proof is exactly the same as for the Theorem 3, since on one hand the action (81) has the same structure as the first order action (32) for the standard dressing cosets, and on the other hand the relation (57) responsible of the gauge invariance of the action (32) still exists and is generalized throught the relation (97) on the domain of $R$.

\section{The gauge invariance of second order ac- tions}

In this section we shall prove the gauge invariance of the second order actions of the types (47) and (95) for all cases of dressing cosets considered in Section 2,3 and 4 . In other words, we shall etablish that the dual models live respectively on the target spaces $F \backslash G$ and $F \backslash \hat{G}$.

We give now a proof of the gauge invariance of the second order actions of the type (47), (95) with respect to the dressing action of $F$ on $G$. Let us examine this dressing action by taking an element $f \in F$ and let it act on any element $l \in D$ by the standard left multiplication. Since $l=g \hat{h}$ with $g \in G$ and $\hat{h} \in \hat{G}$, the left action of $F$ on $D$ can be decomposed as

$$
f l=(g \Delta g)(\Delta \hat{h} \hat{h}) .
$$

Then the dressing action on $G$ is given by $f \triangleright g=g \Delta g$ and on $\hat{G}$ by $f \triangleright \hat{h}=$ $\Delta \hat{h} \hat{h}$. From Eq.(101) we get for the variation $\Delta g$ and $\Delta \hat{h}$ :

$$
\operatorname{Ad}_{g^{-1}} f=\Delta g \Delta \hat{h}
$$

In order to prove the gauge invariance we write the usual expression of the Poisson-Lie models in term of components. Recall that $\mathcal{R}$ is a $n$-dimensional linear subspace of $\mathcal{D}$ and can be written as a linear combinaison of the $2 n$ generators $\left\{t_{i}, T^{j}\right\}$ of $\mathcal{D}$ such that $\left\langle t_{i} \mid T^{j}\right\rangle=\delta_{i}^{j},\left\langle T^{i} \mid T^{j}\right\rangle=\left\langle t_{i} \mid t_{j}\right\rangle=0$ with $t_{i} \in \mathcal{G}$ and $T^{i} \in \hat{\mathcal{G}}$. Let us define the adjoint action of an element $g \in G$ on the generators $\{t, T\}$ of the Lie algebra $\mathcal{D}$ :

$$
\operatorname{Ad}_{g^{-1}} t_{i}=a(g)_{i}^{j} t_{j}, \quad \operatorname{Ad}_{g^{-1}} T^{i}=b(g)^{i j} t_{j}+a^{-1}(g)_{j}^{i} T^{j},
$$

We can express the components of $\Pi^{g}$ in term of the $a(g)$ and $b(g)$ matrices as $\Pi(g)^{i j}=b(g)^{i r} a^{-1}(g)_{r}^{j}$. Then the action (47) becomes:

$$
\mathcal{S}(g)=\frac{1}{2} \int d \xi^{+} d \xi^{-}\left(\partial_{+} g g^{-1}\right)^{i}(R+\Pi(g))_{i j}^{-1}\left(\partial_{-} g g^{-1}\right)^{j}
$$


Let us consider the action (104) evaluated in $g \Delta g$ for a given $f(\tau, \sigma)$ :

$$
\mathcal{S}(g \Delta g)=\mathcal{S}(g)+\Delta \mathcal{S}(g)=\int d \xi^{+} d \xi^{-}(\mathcal{L}(g)+\Delta \mathcal{L}(g)),
$$

with $\mathcal{L}$ the Lagrangian of the action $S(g)$. To express the first order variation $\Delta \mathcal{L}(g)$ we need the infinitesimal variation of $\partial_{ \pm} g g^{-1} \in \mathcal{G}$ and of the PoissonLie bivector $\Pi(g)^{i j}$. Any element $\gamma \in \operatorname{Ker}\left(R+R^{*}\right)$ can be written as $\gamma=$ $\gamma_{\alpha} T^{\alpha}$ where the greek indices refer to $\operatorname{Ker}\left(R+R^{*}\right)=\operatorname{Span}\left(T^{\alpha}\right)$ with $\alpha=$ $1, \ldots, \operatorname{dim} \mathcal{F}$, and the subalgebra can be written $\mathcal{F}=\operatorname{Span}\left(T^{\alpha}+R^{\alpha i} t_{i}\right)$. Thus an element $f$ of $F$ takes the form $f=e^{\epsilon_{\alpha}\left(T^{\alpha}+R^{\alpha i} t_{i}\right)} \in F$, where the parameters $\epsilon_{\alpha}$ are the coordinates of $f$ on the group $F$.

The infinitesimal variations of the vectors $\partial_{ \pm} g g^{-1}$ and of the bivector $\Pi(g)^{i j}$ are given by:

$$
\begin{gathered}
\Delta \partial_{ \pm} g g^{-1}=\epsilon_{\alpha}\left[R^{\alpha i} f_{l i}^{k}-\left[\hat{f}_{l}^{\alpha k}-f_{l s}^{\alpha} \Pi(g)^{s k}\right]\right]\left(\partial_{ \pm} g g^{-1}\right)^{l} t_{k} \\
\Delta \Pi(g)^{i j}=\epsilon_{\alpha}\left[R^{\alpha k}+\Pi(g)^{\alpha k}\right] \nabla_{k} \Pi(g)^{i j}
\end{gathered}
$$

with $\nabla_{k} \Pi(g)^{i j}=-\tilde{f}_{k}^{i j}-f_{k r}^{i} \Pi(g)^{j r}+f_{k r}^{j} \Pi(g)^{i r}, f$ and $\hat{f}$ the structure constants of the Lie algebras $\mathcal{G}$ and $\hat{\mathcal{G}}$ respectively.

Denoting $E(g)=R+\Pi(g)$, the infinitesimal variation of the Lagrangian of the action (104) is:

$$
\begin{aligned}
\Delta \mathcal{L}(g) & =\left(\Delta \partial_{+} g g^{-1}\right)^{i}\left(E(g)^{-1}\right)_{i j}\left(\partial_{-} g g^{-1}\right)^{j}+\left(\partial_{+} g g^{-1}\right)^{i}\left(E(g)^{-1}\right)_{i j}\left(\Delta \partial_{-} g g^{-1}\right)^{j} \\
& -\left(\partial_{+} g g^{-1}\right)^{i}\left(E(g)^{-1} \Delta E(g) E(g)^{-1}\right)_{i j}\left(\partial_{-} g g^{-1}\right)^{j} .
\end{aligned}
$$

Replacing by equalities (106)-(107) and using the following Jacobi identity for $\Pi(g)$ :

$$
f_{s r}^{i} \Pi(g)^{j s} \Pi(g)^{k r}+\tilde{f}_{s}^{i j} \Pi(g)^{k s}+c p(i, j, k)=0 .
$$

Eq.(108) becomes:

$$
\Delta \mathcal{L}(g)=\epsilon_{\alpha}\left(\partial_{+} g g^{-1}\right)^{i}\left(E(g)^{-1}\right)_{i k} \Omega^{\alpha, k l}\left(E(g)^{-1}\right)_{l j}\left(\partial_{-} g g^{-1}\right)^{j},
$$

where

$$
\Omega^{\alpha, k l}=-R^{\alpha s} f_{s t}^{l} R^{k t}+R^{k s} f_{s t}^{\alpha} R^{t l}-R^{\alpha s} f_{s t}^{k} R^{t l}+\hat{f}_{s}^{\alpha l} R^{k s}+\hat{f}_{s}^{k l} R^{\alpha s}+\hat{f}_{s}^{\alpha k} R^{s l} .
$$

The vanishing of $\Omega^{\alpha, k l}$ is the consequence of the relation (57):

$$
J_{A_{f}} \hat{J}+J\left[\operatorname{Ad}_{f}, R\right] \hat{J}=R \operatorname{Ad}_{f} R, \forall f \in F,
$$

for an infinitesimal variation $\delta f$. We here thus proved the invariance of the action (47) under the dressing action of $F$ on $G$, i.e. $\mathcal{S}(g \Delta g)=\mathcal{S}(g), g \in G$. 
Consequently, the target spaces of the dual pair are in fact the cosets $F \backslash G$ and $F \backslash \hat{G}$.

Remark 1: Eq.(57) is not the only set of constraints on $R$, the following one comes from the fact that $\mathcal{F}$ is a Lie subalgebra therefore verifies $[\mathcal{F}, \mathcal{F}] \subset \mathcal{F}$ or equivalently:

$$
\Longleftrightarrow\left\{\begin{array}{l}
\hat{f}_{M}^{\alpha \beta}+R^{\beta s} f_{s M}^{\alpha}-R^{\alpha s} f_{s M}^{\beta}=0 \\
{\left[R^{\alpha S} f_{s t}^{l} R^{\beta t}-R^{\beta s} \hat{f}_{s}^{\alpha l}+R^{\alpha s} \hat{f}_{s}^{\beta l}\right]\left(R^{-1}\right)_{l M}=0}
\end{array}\right.
$$

Remark 2: In the case of the generalized dressing cosets the proof of the gauge invariance is similar. Indeed, if we denote $\hat{\mathcal{A}} \oplus \operatorname{Im} R=\operatorname{Span}\left(t_{M}\right)$ with $M=\operatorname{dim} \operatorname{Ker} \hat{R}+1, \ldots, \operatorname{dim} \mathcal{G}$. Then the action (95) becomes simply:

$$
\mathcal{S}(g)=\frac{1}{2} \int d \xi^{+} d \xi^{-}\left(\partial_{+} g g^{-1}\right)^{M}\left(R+\Pi_{R}(g)\right)_{M N}^{-1}\left(\partial_{-} g g^{-1}\right)^{N}
$$

Since the relations (97) and (98) still hold and the generalized action has the same structure as the standard dressing cosets action, the generalized dressing cosets action is gauge invariant.

\section{Application of generalized dressing cosets: Models of Sfetsos [5]}

Let us show that the models studied by Sfetsos [5] enter in the category of the generalized dressing cosets. Recall from the beginning of Section 4 that the dual pair of the models is encoded in the choice of operators $R$ : $\operatorname{Dom} R \rightarrow \operatorname{Im} R$ and $\hat{R}: \operatorname{Dom} \hat{R} \rightarrow \operatorname{Im} \hat{R}$. Here $\operatorname{Dom} \hat{R}$ and $\operatorname{Im} R$ are subsets of $\mathcal{G}, \operatorname{Dom} R$ and $\operatorname{Im} \hat{R}$ are subsets of $\hat{\mathcal{G}}$, and both operators verify the relations (97) and (98). We consider now a particular case which will turn out to give the Sfetsos models.

1. $\operatorname{Ker} R=\operatorname{Ker}\left(R+R^{*}\right)=\{0\}$

2. $\operatorname{Ker} \hat{R}=\operatorname{Ker}\left(\hat{R}+\hat{R}^{*}\right) \neq\{0\}$ and $\operatorname{Ker} \hat{R}$ is a Lie subalgebra of $\mathcal{G}$.

Because the bilinear form is non degenerate on $\operatorname{Im} R \oplus \operatorname{Im} \hat{R}$, we can choose a basis $\left\{t_{i}, T^{j}\right\}$ of $\mathcal{D}$ such that $\operatorname{Im} R=\operatorname{Span}\left(t_{M}\right), \operatorname{Im} \hat{R}=\operatorname{Span}\left(T^{M}\right), \operatorname{Ker} \hat{R}=$ $\operatorname{Span}\left(t_{\alpha}\right)$, and

$$
\left\langle t_{i} \mid T^{j}\right\rangle=\delta_{i}^{j}, \quad(i, j)=1, \ldots, n .
$$

Here $\alpha=1, \ldots, \operatorname{dim} \mathcal{F}$ and $M=\operatorname{dim} \mathcal{F}+1, \ldots, \operatorname{dim} \mathcal{G}$.

Note in particular, that the condition (113) implies also $\left\langle T^{M} \mid t_{N}\right\rangle=\delta_{N}^{M}$, 
$(M, N)=\operatorname{dim} \mathcal{F}+1, \ldots, \operatorname{dim} \mathcal{G}$. We define the subspace $\mathcal{A}$ of $\hat{\mathcal{G}}$ as $\mathcal{A}=$ $\operatorname{Span}\left(T^{\alpha}\right)$. We note that the points $1-2$ imply that $\mathcal{G}=\operatorname{Dom} \hat{R}=\operatorname{Im} R \oplus$ $\operatorname{Ker} \hat{R}, \hat{\mathcal{G}}=\operatorname{Dom} R=\operatorname{Im} \hat{R} \oplus \mathcal{A}$ and $\mathcal{F}=\operatorname{Ker} \hat{R}$.

The action (95) becomes that of Sfetsos [5]:

$$
\mathcal{S}(g)=\frac{1}{2} \int d \xi^{+} d \xi^{-}\left(\partial_{+} g g^{-1}\right)^{M}\left(R+\Pi_{R}(g)\right)_{M N}^{-1}\left(\partial_{-} g g^{-1}\right)^{N},
$$

where $(O)_{M N}^{-1}$ means the inverse matrix of $\left\langle T^{M} \mid O T^{N}\right\rangle$. The dual theory is obtained by working out the action (96) for our particular choice of $R$ and $\hat{R}$ which gives:

$\mathcal{S}(\hat{g})=\frac{1}{2} \int d \xi^{+} d \xi^{-}\left(\partial_{+} \hat{g} \hat{g}^{-1}\right)_{i}\left(\begin{array}{cc}\left(\hat{R}+\hat{\Pi}_{R}(\hat{g})\right)_{\alpha \beta} & \left(\hat{R}+\hat{\Pi}_{R}(\hat{g})\right)_{\alpha N} \\ \left(\hat{R}+\hat{\Pi}_{R}(\hat{g})\right)_{M \beta} & \left(\hat{R}+\hat{\Pi}_{R}(\hat{g})\right)_{M N}\end{array}\right)^{-1}\left(\partial_{-} \hat{g} \hat{g}^{-1}\right)_{j}$.

Note that the components $\hat{R}_{\alpha \beta}, \hat{R}_{\alpha N}$ and $\hat{R}_{M \beta}$ vanish since $\operatorname{Ker} \hat{R}=\operatorname{Span}\left(t_{\alpha}\right)$, leading to:

$$
\mathcal{S}(\hat{g})=\frac{1}{2} \int d \xi^{+} d \xi^{-}\left(\partial_{+} \hat{g} \hat{g}^{-1}\right)_{i}\left(\begin{array}{cc}
\hat{\Pi}_{R}(\hat{g})_{\alpha \beta} & \hat{\Pi}_{R}(\hat{g})_{\alpha N} \\
\hat{\Pi}_{R}(\hat{g})_{M \beta} & \left(\hat{R}+\hat{\Pi}_{R}(\hat{g})\right)_{M N}
\end{array}\right)^{-1}\left(\partial_{-} \hat{g} \hat{g}^{-1}\right)_{j} .
$$

Furthermore, from $\left.\hat{R}\right|_{\operatorname{Im} R}=\left(\left.R\right|_{\operatorname{Im} \hat{R}}\right)^{-1}$ we obtain $\hat{R}_{M N}=\left(R^{-1}\right)_{M N}$, hence the action (116) becomes that of the dual Sfetsos theory:

$\mathcal{S}(\hat{g})=\frac{1}{2} \int d \xi^{+} d \xi^{-}\left(\partial_{+} \hat{g} \hat{g}^{-1}\right)_{i}\left(\begin{array}{cc}\hat{\Pi}_{R}(\hat{g})_{\alpha \beta} & \hat{\Pi}_{R}(\hat{g})_{\alpha N} \\ \hat{\Pi}_{R}(\hat{g})_{M \beta} & \left(R^{-1}+\hat{\Pi}_{R}(\hat{g})\right)_{M N}\end{array}\right)^{-1}\left(\partial_{-} \hat{g} \hat{g}^{-1}\right)_{j}$.

Following the results of Section 5, Sfetsos'actions (114) and (117) are gauge invariant, if the conditions (97) and (98) are imposed. Note that the gauge invariance of the action (114) was already proved by Sfetsos, however as far as the action (117) is concerned, Sfetsos has only conjectured its gauge invariance. In our paper we have proved his conjecture with the group $F$ acting on $\hat{G}$ in the dressing way described in Eq.(101).

\section{Conclusions and Outlook}

We gave a new algebraic definition of the dual pair of $\sigma$-models introduced in [2] under the name of the dressing cosets. Our new construction has led to a more general class of models than those constructed in [2]. We call them the generalized dressing cosets. To specify more closely the character of the generalization let us make the book-keeping of all the models considered in this paper following the properties of the fundamental operators $R$ and $\hat{R}$. 
Standard Poisson-Lie T-duality [1]: $\operatorname{Ker} R=\operatorname{Ker} \hat{R}=\{0\}$ and $\operatorname{Ker}\left(R+R^{*}\right)=\{0\}$. There is no gauge group in this case.

Standard dressing cosets [2]: $\operatorname{Ker} R=\operatorname{Ker} \hat{R}=\{0\}$ and $\operatorname{Ker}(R+$ $\left.R^{*}\right) \neq\{0\}$. The gauge group is a subgroup of $D$ but neither of $G$ nor of $\hat{G}$.

Sfetsos models [5]: Either $\operatorname{Ker} R=\operatorname{Ker}\left(R+R^{*}\right) \neq\{0\}$ or $\operatorname{Ker} \hat{R}=$ $\operatorname{Ker}\left(\hat{R}+\hat{R}^{*}\right) \neq\{0\}$. The gauge group is a subgroup of $G$ or of $\hat{G}$ respectively.

New Sfetsos-like cases: $\operatorname{Ker} R=\operatorname{Ker}\left(R+R^{*}\right) \neq\{0\}$ and $\operatorname{Ker} \hat{R}=$ $\operatorname{Ker}\left(\hat{R}+\hat{R}^{*}\right) \neq\{0\}$. The gauge group is the direct product of the Lie groups corresponding to the Lie algebras $\operatorname{Ker} R$ and $\operatorname{Ker} \hat{R}$.

Generalized dressing cosets: $\operatorname{Ker} R \neq\{0\}$ and/or $\operatorname{Ker} \hat{R} \neq\{0\}$ and $\operatorname{Ker}\left(R+R^{*}\right) \neq\{0\}$. The gauge group is any kind of subgroup of $D$.

Furthermore we have directly proved the gauge invariance of all second order actions. In the future we plan to study the dressing cosets in the case where the Drinfeld double $D$ is not perfect.

\section{References}

[1] C. Klimčík and P. Ševera, Dual Non-Abelian Duality and the Drinfeld Double, Phys.Lett.B351 (1995) 455-462, hep-th/9502122

[2] C. Klimčík and P. Ševera, Dressing Cosets, Phys.Lett. B381 (1996) 5661, hep-th/9602162

[3] K. Gawȩdzki and A. Kupiainen, Coset Construction from Functional Integrals, Nucl.Phys. B320 (1989) 625

[4] C. Klimčík, Yang-Baxter $\sigma$-models and $d S / A d S$ T-duality, JHEP 0212 (2002) 051, hep-th/0210095

[5] K. Sfetsos, Duality-invariant class of two-dimensional field theories, Nucl.Phys. B561 (1999) 316-340, hep-th/9904188

[6] C. Klimčík and P. Ševera, Poisson-Lie T-duality and Loop Groups of Drinfeld Doubles, Phys.Lett. B372 (1996) 65-71, hep-th/9512040

[7] A.M. Polyakov and P.B. Wiegmann, Goldstone fields in two-dimensions with multivalued actions, Phys. Lett. B141 (1984) 223 